\title{
RESEARCH HIGHLIGHT The hens guarding epithelial cancer fox-houses
}

\author{
Liam T. Gaynor ${ }^{1,2}$ and Ramesh A. Shivdasani $\mathbb{D}^{1,3,4 凶}$ \\ (c) CEMCS, CAS 2021 \\ Cell Research (2022) 32:225-226; https://doi.org/10.1038/s41422-021-00599-2
}

\begin{abstract}
Esophageal micro-tumors that form rapidly in a carcinogeninduced mouse model regress almost as fast. Normalappearing Notch1-mutant clones with their own intrinsic cancer potential competitively extrude these micro-tumors from the epithelium, demonstrating their surprising shortterm protection of the organism.
\end{abstract}

Cancers are presented in the clinic years after a tumor first takes hold. This delay limits our appreciation of how the original cells expand, first into small benign masses and gradually into invasive cancers. The conventional wisdom from human and animal studies is that progression reflects serial accumulation of mutations, though it is unclear what specific advantage each additional mutation confers. Improved understanding of early tumorigenesis could augment strategies for cancer prevention or early detection, with particular impact on epithelial cancers (carcinomas), which account for the highest death tolls.

In cycling epithelia, such as the epidermis and the lining of the digestive tract, native stem cells compete with one another for finite niche support; because wild-type stem cells are functionally equipotent, this physiologic process is called neutral drift. At the onset of cancer, oncogene or tumor suppressor mutations are thought to enhance fitness, allowing a cell to outcompete its wild-type neighbors and hence seed the earliest, benign neoplasms. This clonal advantage can be measured, for example in mouse intestinal stem cells carrying the same Apc, Kras or Trp53 mutations that drive human intestinal adenomas. ${ }^{1}$ Given that intestinal stem cells divide frequently, one might expect fixed mutant clones to quickly engender sizable masses. Stem cell (and by extension adenoma) growth is, however, restrained by physical and niche constraints, and although mutant intestinal crypts influence their neighbors' physiology, nearby crypts appear ostensibly normal. ${ }^{2}$ Wild-type and mutant crypts thus co-exist with seemingly limited conflict and up to $1 \%$ of colonic crypts in 50-60-year-old people bear potential oncogenic mutations without overt pathology; fewer than 1 in 3 million such mutant crypts may spawn cancer in a person's lifetime. ${ }^{3}$

Other epithelia replicate this minuscule probability. At least a quarter of superficially normal adult airway (in smokers), skin, and esophageal surfaces carry driver oncogene mutations, ${ }^{4-6}$ far greater than the proportion of tumors arising in those sites. Thus, driver mutations do not alone generate tumors. Although mutant clones in some tissues occupy increasingly larger areas as people and mice age, ${ }^{5-7}$ their relative advantage over wild-type clones, if any, is dictated by the fitness of nearby cells, and cells of similar fitness drift neutrally. Lesions that distort the mouse epidermis quickly regress ${ }^{8}$ and in former smokers, wild-type bronchial clones may over time supplant mutant clones. ${ }^{6}$ In all tissues, cells must sustain additional genetic alterations to turn cancerous. While investigating this mutational progression, the authors of a recent report in Nature discovered an unexpected protective element of epithelial clone dynamics. ${ }^{9}$

Because smoking is a risk factor for esophageal squamous cell cancer, Colom et al. investigated mouse esophageal tumor evolution by adding diethylnitrosamine (DEN), a mutagen found in tobacco smoke, to the drinking water for a short period. ${ }^{9}$ Within 10 days after DEN withdrawal, random mutagenesis had led to formation of hundreds of small dysmorphic KRT6-expressing neoplasms, but most "micro-tumors" regressed within 3 months; few large tumors remained at 1 year and squamous cancers appeared by 18 months. Polyclonal 10-day-old lesions showed positive selection for Notch1 and Trp53 mutations, while 12month-old tumors were monoclonal, lacked genome stability, and showed positive selection for Atp2a2, Notch1, Notch2, Chuk, and Adam 10 mutations. ${ }^{9}$

The authors carefully excluded growth disadvantage, apoptosis, and immune surveillance as causes of micro-tumor attrition. A high density of Notch 1 mutations in the tissue near micro-tumors then led them to wonder whether Notch1-mutant "normal" clones, i.e., not manifesting as $\mathrm{KRT}^{+}$micro-tumors, may curtail nearby neoplasia by virtue of greater fitness. To test this hypothesis, they mimicked Notch1 mutation, or lack of Notch signaling, by forcibly expressing a dominant-negative (DN) form of the Notch effector protein MAML1. Micro-tumor loss was enhanced as a result, in proportion to the surface area of Notch-inhibited cells, indicating clonal competition, and microscopy revealed extrusion of micro-tumors encircled by DN-MAML1-expressing esophageal cells (Fig. 1). Conversely, the authors reasoned that Notch inhibition in tumors might mitigate their competitive disadvantage, rendering them neutral with respect to DN-MAML1 cells. Indeed, pharmacologic Notch inhibition after micro-tumor formation allowed those growths to persist. ${ }^{9}$

Through sound experimental models, deep DNA sequencing, and mechanism-based perturbation, this study uncovers unexpected pruning of early neoplasms as a result of competition from surrounding Notch1-mutant clones. Thus, until the latter clones might themselves sustain additional mutations and form cancers, their relative fitness appears to guard the tissue at large from that ongoing risk (Fig. 1). The brief clonal advantage of Notch1-mutant esophageal cells ${ }^{7}$ may derive in part from this salutary effect of eliminating fledgling tumors, which further helps explain the low esophageal tumor incidence relative to the abundance of mutant clones. Although tumors that thrived at 1 year by evading this

${ }^{1}$ Department of Medical Oncology, Dana-Farber Cancer Institute, Boston, MA, USA. ${ }^{2}$ Graduate Program in Biological \& Biomedical Sciences, Harvard Medical School, Boston, MA,

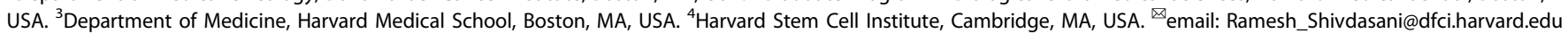




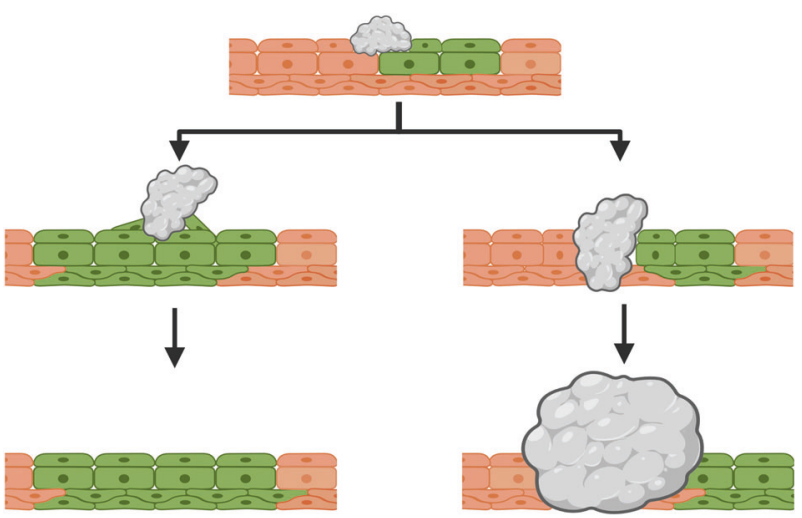

Fig. 1 In continuous competition for the basal layer in stratified esophageal epithelium, micro-tumors (grey) interface with wildtype (orange, disadvantaged) and other mutant (green, selectively advantaged) clones. Micro-tumors that collide with the latter cells get extruded from the epithelium. Only lesions that survive this competition and accumulate additional mutations form invasive cancers. Graphics generated in Biorender.

protective mechanism differed in mutational profile from 10-dayold micro-tumors, it is unclear whether the sum of mutations selected in the interim conferred specific counter-advantage over Notch1-mutant cells or reflected processes unrelated to clonal competition.

Contests for limited space in each tissue follow individual niche constraints. In contrast to stratified esophageal and skin epithelia, intestinal stem cells are restrained within distinct crypt structures and Apc-null stem cells outcompete their wild-type siblings in part by inhibiting local Wnt signals. ${ }^{10,11}$ The columnar intestinal epithelium is not subject to the extrusion mechanism reported in squamous esophageal mucosa ${ }^{9}$ or skin. ${ }^{8}$ This may be one material difference in tissue clonal dynamics; also still unknown is whether tumors are competitively extruded from human and other mouse stratified epithelia.

Because advanced cancers are rarely curable, freedom from cancer in the long run depends heavily on prevention, early detection, and better understanding of pre- and early-neoplastic lesions. Further investigation of aging human tissues, coupled with precise tracing of early tumors in animal models, will clarify how natural safeguards or clinical interventions may thwart incipient cancers in different sites.

\section{REFERENCES}

1. Vermeulen, L. et al. Science 342, 995-998 (2013).

2. Yum, M. K. et al. Nature 594, 442-447 (2021).

3. Lee-Six, H. et al. Nature 574, 532-537 (2019).

4. Martincorena, I. et al. Science 348, 880-886 (2015).

5. Martincorena, I. et al. Science 362, 911-917 (2018).

6. Yoshida, K. et al. Nature 578, 266-272 (2020).

7. Colom, B. et al. Nat. Genet. 52, 604-614 (2020).

8. Brown, S. et al. Nature 548, 334-337 (2017).

9. Colom, B. et al. Nature 598, 510-514 (2021).

10. Flanagan, D. J. et al. Nature 594, 430-435 (2021).

11. van Neerven, S. M. et al. Nature 594, 436-441 (2021).

\section{COMPETING INTERESTS}

The authors declare no competing interests.

\section{ADDITIONAL INFORMATION}

Correspondence and requests for materials should be addressed to Ramesh $\mathrm{A}$. Shivdasani.

Reprints and permission information is available at http://www.nature.com/ reprints 